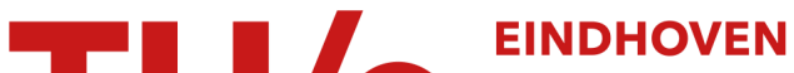 \\ UNIVERSITY OF \\ TECHNOLOGY
}

\section{Robust optimal sensor planning for occlusion handling in dynamic robotic environments}

Citation for published version (APA):

Mohan, R., \& de Jager, B. (2019). Robust optimal sensor planning for occlusion handling in dynamic robotic environments. IEEE Sensors Journal, 19(11), 4259-4270. [8643386].

https://doi.org/10.1109/JSEN.2019.2899929

DOI:

10.1109/JSEN.2019.2899929

Document status and date:

Published: 01/06/2019

Document Version:

Accepted manuscript including changes made at the peer-review stage

Please check the document version of this publication:

- A submitted manuscript is the version of the article upon submission and before peer-review. There can be important differences between the submitted version and the official published version of record. People interested in the research are advised to contact the author for the final version of the publication, or visit the $\mathrm{DOI}$ to the publisher's website.

- The final author version and the galley proof are versions of the publication after peer review.

- The final published version features the final layout of the paper including the volume, issue and page numbers.

Link to publication

\section{General rights}

Copyright and moral rights for the publications made accessible in the public portal are retained by the authors and/or other copyright owners and it is a condition of accessing publications that users recognise and abide by the legal requirements associated with these rights.

- Users may download and print one copy of any publication from the public portal for the purpose of private study or research.

- You may not further distribute the material or use it for any profit-making activity or commercial gain

- You may freely distribute the URL identifying the publication in the public portal.

If the publication is distributed under the terms of Article $25 \mathrm{fa}$ of the Dutch Copyright Act, indicated by the "Taverne" license above, please follow below link for the End User Agreement:

www.tue.nl/taverne

Take down policy

If you believe that this document breaches copyright please contact us at:

openaccess@tue.nl

providing details and we will investigate your claim. 


\title{
Robust Optimal Sensor Planning for Occlusion Handling in Dynamic Robotic Environments
}

\author{
Rishi Mohan and Bram de Jager
}

\begin{abstract}
Optimal sensor planning for workspace detection in robotic environments is hindered due to sensor occlusions. These occlusions are often dynamic. Probabilistic optimization frameworks, which generally deal with the uncertain nature of these occlusions suffer from unreliability and/or unavailability of probability distribution functions. This paper proposes and analyses a robust optimization approach (minimax) which generates sensor configurations based on occlusion scenarios that cause maximum obstruction of the robotic workspace. The optimal solution is independent of probability distribution functions and provides a guaranteed level of workspace visibility regardless of occluder positions, thus accounting for random occlusions. The method also allows the user to determine the impact of the worst-case occlusion scenarios leading to a broader perspective on sensor planning. Evaluation of the approach for a mobile medical $X$-ray robotic system in a simulation healthcare environment shows the effectiveness of the proposed method.
\end{abstract}

Index Terms-Optimal Sensor Placement, Minimax Optimization, Workspace Detection, Dynamic Occlusions

\section{INTRODUCTION}

$\mathbf{P}$ OPULATED human-robot environments such as hospitals, museums, production shop floors, etc., primarily rely on a network of multiple sensors for detecting a target workspace. Consequently, planning the optimal location and orientation of different sensors in the target environment is an important design problem as it impacts the performance and cost of the network [1]. A particular sensor configuration (location and orientation) defines the size and shape of the coverage area, thereby directly impacting the network performance in terms of the amount of space observed by the network. Additionally, sensor costs and installation constraints prevent utilizing a large number of sensors in an environment [2]. Sensor planning of the limited number of sensors is thus formulated as an off-line constrained optimization problem which aims to maximize the coverage potential while accounting for location and task specific constraints [3].

Determining the optimal sensor configuration for multisensor networks is only a partially resolved problem [4]. One of the major challenges in sensor planning is accounting for dynamic occlusions due to a changing environment [5], [6]. In human-robot environments, an occlusion occurs when a sensor (such as a ceiling mounted camera) loses sight of the target workspace due to obstruction by objects, human beings or the robot itself [7]. Due to the constantly changing environment, these occlusions are dynamic. They cause random loss of

Rishi Mohan and Bram de Jager are with the Department of Mechanical Engineering, Control Systems Technology Section, Eindhoven University of Technology, 5600 MB Eindhoven, The Netherlands.

Corresponding Author: Rishi Mohan (r.mohan@tue.nl) object capture from the sensors and are problematic since their inherent nature is unpredictable and random. The sensor planning problem in the presence of these random occlusions is thus formulated using decision theory, i.e., to determine an optimal configuration (decision) in the presence of inaccessible or inaccurate information on occluder behaviour (uncertainty) [8].

This paper addresses the uncertainty associated with random occlusions in the sensor planning optimization problem. The main contribution is the proposal and analysis of a nonprobabilistic optimization approach that explicitly accounts for the non-deterministic nature of dynamic occlusion without the need for probability distributions. Moreover, it promises a certain guarantee on sensor coverage irrespective of occluder locations. The approach determines an optimal sensor configuration by considering occluder positions that cause maximum occlusion of the target workspace. Such an approach is termed as minimax, where the aim is to optimize the worst-case performance with respect to uncertainties [9]. The focus lies on sensor planning of static networks employed to detect a robotic workspace where dynamic objects appear randomly to occlude the sensors.

The rest of this paper is organized as follows. Section II provides details of associated literature and the main motivation behind this work. The optimization framework along with minimax optimization is discussed in Section III. Section IV provides a numerical validation of the proposed optimization approach. Evaluation of the methodology is illustrated in Section V using a simulation environment. Section VI is dedicated to concluding remarks.

\section{BACKGROUND AND MOTIVATION}

\section{A. Prior Work}

Sensor planning for visual coverage is an exhaustively studied concept and can be regarded as fundamentally stemming from the Art Gallery Problem (AGP) [10]. The AGP provides the solution to finding the minimum number of omnidirectional sensors that can completely observe a given 2D space [11]. There are multiple extensions to the AGP for different applications, however these are restricted in terms of accounting for dynamic occlusions in a 3D space. A detailed account of these extensions and related shortcomings can be found in [12-16] and references therein.

To deal with the non-deterministic, unpredictable nature of dynamic occlusions, the majority of existing research employs a probabilistic framework [14-17]. Obtaining an optimal sensor configuration $\left(\mathbf{S}_{\text {prob }}^{*}\right)$ thus involves minimizing the expected value of a probabilistic cost function 


$$
\mathbf{S}_{\text {prob }}^{*}=\underset{\mathbf{S} \in \mathcal{S}}{\operatorname{argmin}} \mathbb{E}\left[N V\left(\mathcal{O}_{\mu}, \mathbf{S}\right)\right]
$$

where $N V$ is the occluded workspace, $\mathcal{S}$ is the space of all sensor configurations and $\mu$ is the probability distribution function defining the set of positions of dynamic occluders $(\mathcal{O})$ around the workspace.

Apart from probabilistic approaches, a deterministic worstcase approach to sensor planning has also been considered. A necessary and sufficient condition on the number of sensors to guarantee visibility considering worst-case occluder positions is proposed in [14]. The occluding objects are treated as point objects and the visibility of the sensors is based on the ability to observe a certain point $O$ in space or on the object. The visibility analysis states that $n>k$ is the minimum number of sensors $(n)$ required in the presence of $k$ occluding objects to guarantee visibility of $O$ from at least one sensor. The analysis is limited to point objects in 2D and analysis is not treated as a sensor planning problem accounting for constraints on the number and configuration of sensors.

Worst-case occluder positions has also been addressed in [15], which introduces a sensor configuration quality metric based on the likelihood of dynamic occlusion. The visibility of the sensors is considered for a target point or a particular feature point on a target. The expected probability of occlusion of this target point is computed by drawing a subset of occluder positions from a given probability density function of occluder positions. To this end, only the worst-case occluder positions are sampled, which are assumed to be the locations that are very close to the target point. Again, the analysis in [15] is limited to point objects and analysis is provided in 2D space while it is also possible that the worst-case occlusion occurs when the occluder is close to the sensor instead of only the target point.

\section{B. Motivation and Contribution}

For probabilistic sensor planning as given in (1), the probability distribution function $(\mu)$ of occluder positions around the workspace is the overarching defining factor. While this methodology works well in literature for surveillance and tracking tasks, the following limiting observations can be drawn:

1) These works either assume that $\mu$ is known apriori [16], [17] or is approximated from measured historical data [14]. In practical applications, however, the involved distributions are seldom known exactly [18], [19] making probabilistic frameworks vulnerable to the unavailability of $\mu$.

2) The accuracy of the optimal solutions of probabilistic optimization problems is only as good as the accuracy of the distribution functions utilized. In the case of unavailable distributions the data is generated by subjective judgement [18]. This might prove to be highly inaccurate.

Based on the these observations, this work proposes a robust (minimax) approach to sensor planning problem. Minimax is a non-probabilistic robust optimization method that stems from game theory, where an optimal minimizing strategy is developed against an adversary that maximizes your loss [20], [21]. An optimal sensor configuration $\left(\mathbf{S}_{\text {minimax }}^{*}\right)$ is determined by considering occluder positions $(\mathcal{O})$ that potentially try to maximize the occluded robotic workspace $(N V)$.

$$
\mathbf{S}_{\text {minimax }}^{*}=\arg \min _{\mathbf{S} \in \mathcal{S}} \max _{\mathbf{O} \in \mathcal{O}} N V(\mathbf{O}, \mathbf{S})
$$

Minimax is independent of probability distribution, thereby making it free of the unreliability or unavailability of a distribution function. Moreover, probabilistic solutions are only feasible for the "average" case and may prove to be poor against specific (critical) scenarios [14], [22]. Design of minimax sensor configurations is robust to effects of random occlusions since all possible scenarios (under user-specified constraints) are considered.

The main contributions of this work are:

1) In the absence of trustworthy probability distribution functions on occluder scenarios, this work provides a non-probabilistic robust optimization approach to sensor planning for applications which encounter random occluding objects.

2) Optimal sensor configurations obtained from the proposed approach ensure a certain guarantee on workspace visibility regardless of locations and positions of randomly occurring occlusions.

3) The visibility analysis and optimal planning of a multisensor network is performed in three dimensional $\left(\mathbb{R}^{3}\right)$ space as opposed to analysis in $\mathbb{R}^{2}$ in previous works which treat occlusions [14], [15]. This provides a more realistic solution for applications like workspace detection in 3D environments.

The robust optimization approach is evaluated using a simulation environment in which the robotic workspace and moving objects are modelled as polyhedra in $\mathbb{R}^{3}$. Analysis of the approach is provided by considering a medical X-ray system as the mobile robot and the simulation environment mimics the dynamically changing behaviour of the hospital room in which the robotic X-ray system operates.

\section{Caveats to Minimax Optimization}

1) Conservativeness: It is important to note that minimax optimization solutions are conservative since all emphasis is placed on guarding against the worst possible case [8]. However, for critical applications such as collision detection it is far more important to consider the worst-case scenario such that a collision does not go undetected. Moreover, the proposed approach is also an augmentation to the existing probabilistic frameworks since probability or range of uncertainty can easily be integrated within minimax [23], [24].

2) Computational Demands: Minimax optimization problems are generally known to be computationally demanding [25], [26]. Moreover, sensor planning optimization results in non-convex objective functions. Under these difficulties, researchers opt for metaheuristic search algorithms to effectively solve these problems [27]. This paper also resorts to an existing heuristic algorithm to solve the minimax sensor planning problem. It should be noted that faster and/or tailored 
algorithms for the sensor planning optimization problem exist [28], however that is not the main focus of this paper. In general, the concept of robust optimization proposed in this work is independent of any specific optimization algorithm and this choice is left to the user.

\section{OPTIMIZATION FRAMEWORK}

This section provides basic definitions of elements that constitute the robust optimization framework. The definitions are presented in a generalized manner, such that they are not overly restricted to specific classes of robotic systems or occluder types. All definitions are provided with reference to Fig. 1 and are developed in three dimensions $\left(\mathbb{R}^{3}\right)$.

\section{A. Environment Model}

The general space in which humans and robots co-exist for a considered application (e.g. museum hall, hospital room, etc.) is defined by $\mathcal{E} \subset \mathbb{R}^{3}$. $\mathcal{E}$ contains all objects, including the robotic manipulator, static and dynamic objects and the set of all possible sensor locations.

Definition III.1 (Convex Polyhedron). A convex polyhedron $\mathcal{P}$ is a set of points obtained by the intersection of a finite number of closed half-spaces [29].

$$
\mathcal{P}:=\left\{\gamma \in \mathbb{R}^{3} \mid A \gamma \leq b\right\}
$$

where $A \in \mathbb{R}^{m \times 3}$ and $b \in \mathbb{R}^{m}$ for a polyhedron with $m$ faces.

Definition III.2 (Non-convex Polyhedron). A non-convex polyhedron is the union of a finite number of convex polyhedra such that the union cannot be represented as (3) [30].

The environment model consists of the following elements.

1) Robotic Workspace: The robotic workspace $(\mathcal{W})$ is the target space which should be detected. The workspace is represented as a combination of $n_{w}$ convex polyhedral spaces $\mathbf{W}_{i} \subseteq \mathcal{E}, i \in\left\{1,2, \ldots, n_{w}\right\}$ which represent the space in which the robotic manipulator operates. A combination of these workspaces forms the primary workspace $\mathcal{W}$.

$$
\mathcal{W}=\bigcup_{i=1}^{n_{w}} \mathbf{W}_{i}
$$

The shape, size and location of $\mathcal{W}$ changes according to the operating condition of the manipulator.

2) Robotic Manipulator: The body of the robotic manipulator is modelled as a (non)-convex polyhedron $(\mathcal{R})$ and is a combination of $n_{r}$ convex polyhedra $\mathbf{R}_{j} \subset \mathcal{E}, j \in$ $\left\{1,2, \ldots, n_{r}\right\}$. This combination facilitates flexibility in the construction and simulation of varying body shapes and their movements.

$$
\mathcal{R}=\bigcup_{j=1}^{n_{r}} \mathbf{R}_{j}
$$

Remark. The manipulator is constrained from containing disjoint point sets.

$$
\begin{gathered}
\forall \mathbf{R}_{k} \subset \mathcal{R}, \exists \mathbf{R}_{l} \subset \mathcal{R} \text { such that } \mathbf{R}_{k} \cap \mathbf{R}_{l} \neq \varnothing \\
k, l \in\left\{1,2, \ldots, n_{r}\right\}, k \neq l
\end{gathered}
$$

Remark. $n_{r}$ is not necessarily the number of links of a robotic manipulator.

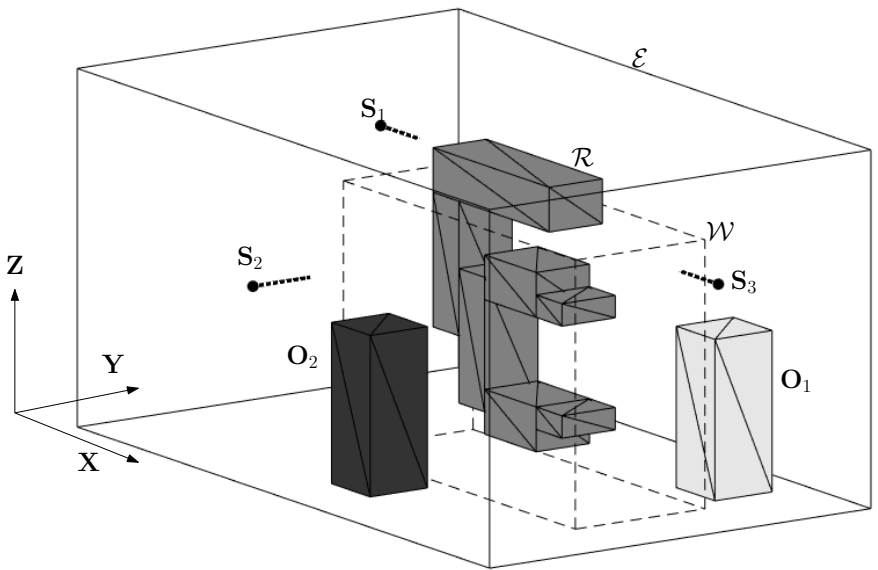

Fig. 1. Generalized representation of the human-robot environment $\mathcal{E}$, which contains the robot workspace $(\mathcal{W})$, the robot body $(\mathcal{R})$, occluder configuration $\hat{\mathbf{O}}=\mathbf{O}_{1} \cup \mathbf{O}_{2}$ and sensor configuration $\hat{\mathbf{S}}=\left[\begin{array}{lll}\mathbf{S}_{1} & \mathbf{S}_{2} & \mathbf{S}_{3}\end{array}\right]^{T}$

3) Occluding Objects: Apart from the robotic manipulator, other objects which reside in $\mathcal{E}$ can act as occluders for a particular sensor. The number of occluders $\left(n_{o}\right)$ present in the environment depends on the specific application at hand. Each occluding object $\mathbf{O}_{k} \subset \mathcal{E}, k \in\left\{1,2, \ldots, n_{o}\right\}$ is modelled as a (non)-convex polyhedron and is permitted to exist in a (userdefined) polyhedral space $\mathcal{C}_{k} \subseteq \mathcal{E}$, i.e., $\mathbf{O}_{k}$ is allowed to move anywhere in $\mathcal{C}_{k}$.

At any instant, the combination of $n_{o}$ occluding objects and their specific locations in $\mathcal{C}_{k}$ at that instant forms a particular occluder configuration $\hat{\mathbf{O}}$.

$$
\hat{\mathbf{O}}=\bigcup_{k=1}^{n_{o}} \mathbf{O}_{k}
$$

where each $\mathbf{O}_{k}$ is present at some particular location within its respective constraint space $\mathcal{C}_{k}$.

All possible occluder configurations are defined by the set $\mathcal{O}$, i.e, $\hat{\mathbf{O}} \in \mathcal{O}$. The set $\mathcal{O}$ forms the various scenarios for the minimax optimization problem (2) from which one or more will be recognized as the worst-case.

Remark. Occluders are non-overlapping with each other and with the manipulator body.

$$
\begin{gathered}
\forall g, h \in\left\{1, \ldots, n_{o}\right\}: g \neq h \Longrightarrow \mathbf{O}_{g} \cap \mathbf{O}_{h}=\varnothing \\
\forall k \in\left\{1,2, \ldots, n_{o}\right\}, \mathbf{O}_{k} \cap \mathcal{R}=\varnothing
\end{gathered}
$$

Remark. The robot manipulator can also be considered as a part of occluding objects if it obstructs a part of the workspace for a particular sensor.

\section{B. Sensor Configuration Model}

The sensor configuration model contains the set of all possible sensor configurations, the user-defined constraints on these configurations and the available sensor types along with their respective intrinsic parameters (e.g. depth, field of view, accuracy, etc.). 


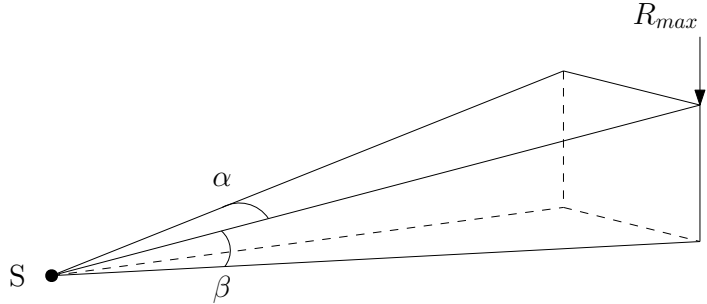

Fig. 2. Pyramid of vision $\left(\mathbf{P} \mathbf{V}_{q}\right)$ for sensor $\mathbf{S}_{q}$ with maximum range $\left(R_{\max }\right)$ and field of view (horizontal $\alpha$, vertical $\beta$ ).

There exist $n_{s}$ sensors in a sensor configuration. Let each sensor $\mathbf{S}_{q} \in \mathbb{R}^{1 \times n_{p}}, q \in\left\{1,2 \ldots, n_{s}\right\}$ be modelled by $n_{p}$ parameters which include (but are not restricted to) the sensor location in $\mathbb{R}^{3}$, pan and tilt angles, etc.

$$
\mathbf{S}_{q}=\left[\begin{array}{llll}
p_{1} & p_{2} & \ldots & p_{n_{p}}
\end{array}\right]
$$

A combination of $n_{s}$ sensors and a particular value of their parameters forms a sensor configuration $\hat{\mathbf{S}} \in \mathbb{R}^{n_{s} \times n_{p}}$.

$$
\hat{\mathbf{S}}=\left[\begin{array}{llll}
\mathbf{S}_{1} & \mathbf{S}_{2} & \ldots & \mathbf{S}_{n_{s}}
\end{array}\right]^{T}
$$

Depending on user-defined constraints, the parameters of each sensor can be varied. Consequently, multiple sensor configurations can be formulated which reside in set $\mathcal{S}$, i.e., $\hat{\mathbf{S}} \in \mathcal{S}$. An optimal sensor configuration $\hat{\mathbf{S}}^{*}$ will be obtained from this set $\mathcal{S}$ as the solution to the optimization problem (2).

\section{Visibility Model}

Given a sensor configuration $\hat{\mathbf{S}}$, occluder configuration $\hat{\mathbf{O}}$ and a robotic workspace $\mathcal{W}$, the amount of workspace visible to the sensor configuration is based on boolean operations on polyhedra (intersection, union, difference, etc.).

The following definitions are introduced for the analysis of workspace visibility:

Definition III.3 (Viewing Pyramid). The viewing pyramid $\left(\mathbf{P V}_{q}\right)$ of a sensor $\mathbf{S}_{q}$ in a sensor configuration $\hat{\mathbf{S}}$ is the maximum possible space that can be observed by the sensor. $P V_{q}$ depends on the range and field of view (FoV) of a sensor and is modelled as a regular pyramid [31] (Fig. 2). The apex of the pyramid is the centre of the sensor, while the height is limited by maximum range $\left(R_{\max }\right)$. The maximum possible space observed by a sensor configuration $\hat{\mathbf{S}}$ is thus defined as

$$
\mathcal{P V}=\bigcup_{q=1}^{n_{s}} \mathbf{P} \mathbf{V}_{q}, \mathcal{P} \mathcal{V} \subset \mathcal{E}
$$

The location and orientation of any $\mathbf{P V}_{q}$ in $\mathcal{E}$ depends on the sensor location and the pan and tilt angle values of the sensor.

Remark. It is possible for the union $\mathcal{P} \mathcal{V}$ to be disjoint.

Definition III.4 (Blind Space). The set of all points in $\mathcal{W}$ which are not in the range and/or field of view of any sensor in $\hat{\mathbf{S}}$ is defined as the blind space $(\mathcal{B})$.

$$
\mathcal{B}=\mathcal{W} \backslash \mathcal{P} \mathcal{V}
$$

Definition III.5 (Occluded Space). The set of points in $\mathcal{W}$ which are occluded by the face(s) of a polyhedral object ( $\hat{\mathbf{O}}$ and/or $\mathcal{R}$ ) for all sensors in a particular sensor configuration is defined as occluded space $(\mathcal{H})$.

$$
\begin{aligned}
& \mathcal{X}=\bigcup_{q=1}^{n_{s}} \bigcup_{k=1}^{n_{o}} \mathcal{M}_{q k} \\
& \mathcal{Y}_{q}=\mathbf{P V}_{q} \backslash \bigcup_{k=1}^{n_{o}} \mathcal{M}_{q k} \\
& \mathcal{Z}=\bigcup_{q=1}^{n_{s}} \mathcal{Y}_{q} \\
& \mathcal{H}=\mathcal{X} \backslash \mathcal{Z}
\end{aligned}
$$

where $\mathcal{M}_{q k}$ is the space occluded by an object $\mathbf{O}_{k}$ for sensor $\mathbf{S}_{q}$ and $\mathcal{Y}_{q}$ is the non-occluded FoV of sensor $\mathbf{S}_{q}$. An illustrative example to compute $\mathcal{H}$ is provided in Fig. 3.

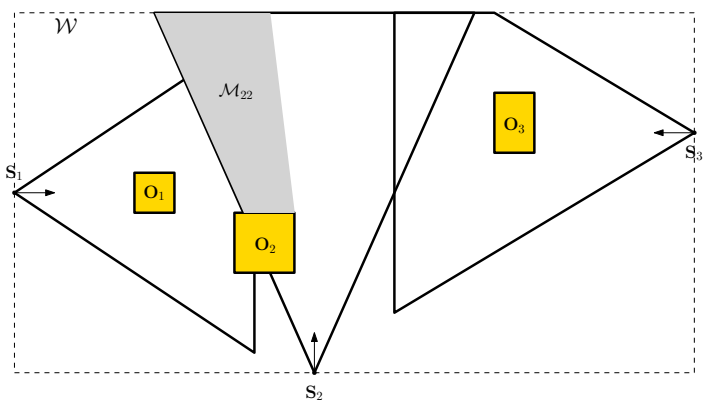

(a) Occluded Space $\left(\mathcal{M}_{22}\right)$ for Sensor $\mathbf{S}_{2}$ (grey). $\mathcal{M}_{21}=$ $\mathcal{M}_{23}=\varnothing$

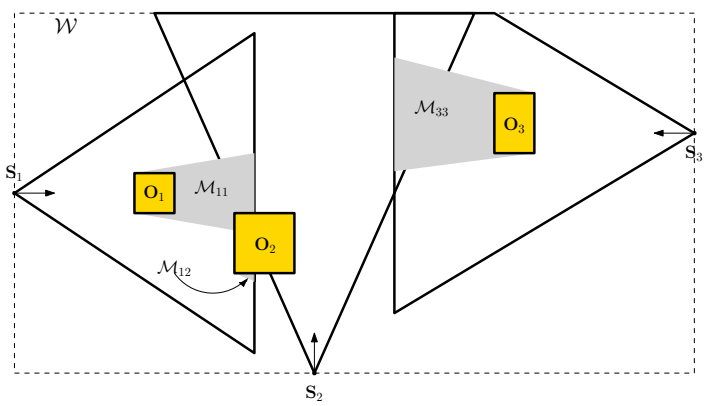

(b) Occluded Space for Sensor $\mathbf{S}_{1}$ and $\mathbf{S}_{2}$ (grey). $\mathcal{M}_{13}=$ $\mathcal{M}_{31}=\mathcal{M}_{32}=\varnothing$

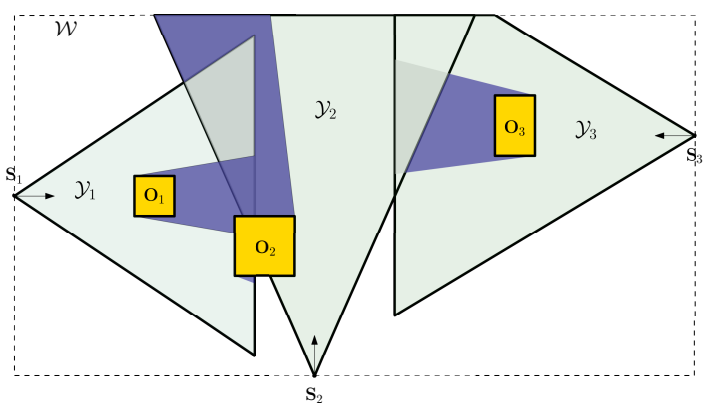

(c) Computing Ocluded Space $\mathcal{H}$ (blue) according to Eq. (14). Non-occluded FoV of sensors $\left(\mathcal{Y}_{q}\right)$ presented in green.

Fig. 3. Illustrative Example - Occluded Space $(\mathcal{H})$ Computation for $n_{s}=3$ sensors and $n_{o}=3$ occluding objects (yellow). 
Based on the visibility analysis explained above, the fraction of workspace not visible to the sensor network $(N V(\hat{\mathbf{O}}, \hat{\mathbf{S}}))$ is the ratio of the volume of the blind and occluded space $(\mathcal{H} \cup \mathcal{B})$ to the volume of $\mathcal{W}$ not occupied by any objects.

$$
N V(\hat{\mathbf{O}}, \hat{\mathbf{S}})=\frac{\operatorname{Vol}(\mathcal{H} \cup \mathcal{B})}{\operatorname{Vol}(\mathcal{W} \backslash(\hat{\mathbf{O}} \cup \mathcal{R}))}
$$

\section{Sensor Planning - Minimax Optimization}

The theory presented previously discusses a function or metric which is a measure of the target space (non-)visibility for a certain sensor configuration, given a specific location of the occluders. As presented in (2), sensor planning in the presence of dynamic occlusions is considered as a robust optimization problem (minimax). To this end, the cost function for the problem is the metric ( $N V)$ given in (15). The minimax optimization problem is defined as

$$
\hat{\mathbf{S}}^{*}=\arg \min _{\hat{\mathbf{S}} \in \mathcal{S}} \max _{\hat{\mathbf{O}} \in \mathcal{O}} N V(\hat{\mathbf{O}}, \hat{\mathbf{S}})
$$

The optimal sensor configuration $\hat{\mathbf{S}}^{*}$ minimizes the maximum $N V$ (worst-case) over the set of all possible occluder configurations $\mathcal{O}$. The elements of the set $\mathcal{O}$ are treated as uncertainty and elements of set $\mathcal{S}$ are the design variables which aim to provide a robust optimum sensor configuration. For each $\hat{\mathbf{S}} \in \mathcal{S}, \exists \hat{\mathbf{O}} \in \mathcal{O}$ such that $N V(\hat{\mathbf{O}}, \hat{\mathbf{S}})=$ $\max (N V(\mathcal{O}, \hat{\mathbf{S}}))$. This is identified as the worst case scenario for $\hat{\mathbf{S}}$. The optimization tries to find $\hat{\mathbf{S}}$ for which this worstcase value is minimum over all $\mathcal{S}$.

A sensor configuration $\hat{\mathbf{S}}^{*}$ and an occluder configuration $\hat{\mathbf{O}}^{*}$ are said to constitute the minimax robust optimal solution if, for $\hat{\mathbf{O}}^{*}$, any configuration other than $\hat{\mathbf{S}}^{*}$ produces a higher $N V$. Additionally, for $\hat{\mathbf{S}}^{*}$, any occluder configuration different from $\hat{\mathbf{O}}^{*}$ gives lower $N V$, i.e., $\hat{\mathbf{S}}^{*}$ has its worst performance in the presence of $\hat{\mathbf{O}}^{*}$.

For the purpose of implementation, this work converts the problem in (16) into the following bi-level optimization problem.

$$
\begin{array}{cl}
\hat{\mathbf{S}}^{*}=\underset{\hat{\mathbf{S}} \in \mathcal{S}}{\arg \min } & N V\left(\hat{\mathbf{O}}^{*}, \hat{\mathbf{S}}\right) \\
\text { s.t. } & l_{m} \leq p_{m} \leq u_{m}, \forall m \in\left\{1, \ldots, n_{p}\right\} \\
& \hat{\mathbf{O}}^{*}=\underset{\hat{\mathbf{O}} \in \mathcal{O}}{\arg \max } N V(\hat{\mathbf{O}}, \hat{\mathbf{S}}) \\
& \text { s.t. } \mathbf{O}_{k} \subseteq \mathcal{C}_{k}, \forall k \in\left\{1, \ldots, n_{o}\right\}
\end{array}
$$

where $l_{m}$ ( respectively $u_{m}$ ) is the lower (respectively upper) bound on each sensor parameter $p_{m}$ and each individual occluder $\mathbf{O}_{k} \in \hat{\mathbf{O}}$ is constrained in the polyhedral space $\mathcal{C}_{k}$.

Minimax problems, in general, have a global optimum termed as the saddle-value by considering concave-convex (or convex-concave) objective functions [32]. The minimization is computed over the convex part and maximization takes place over the concave argument. However, this only holds for cases where the concave-convex function is defined by the product of convex sets [33]. Solving the optimization problem of the form (17) is non-trivial as both the upper (minimization) and lower level (maximization) optimization problems have non-convex

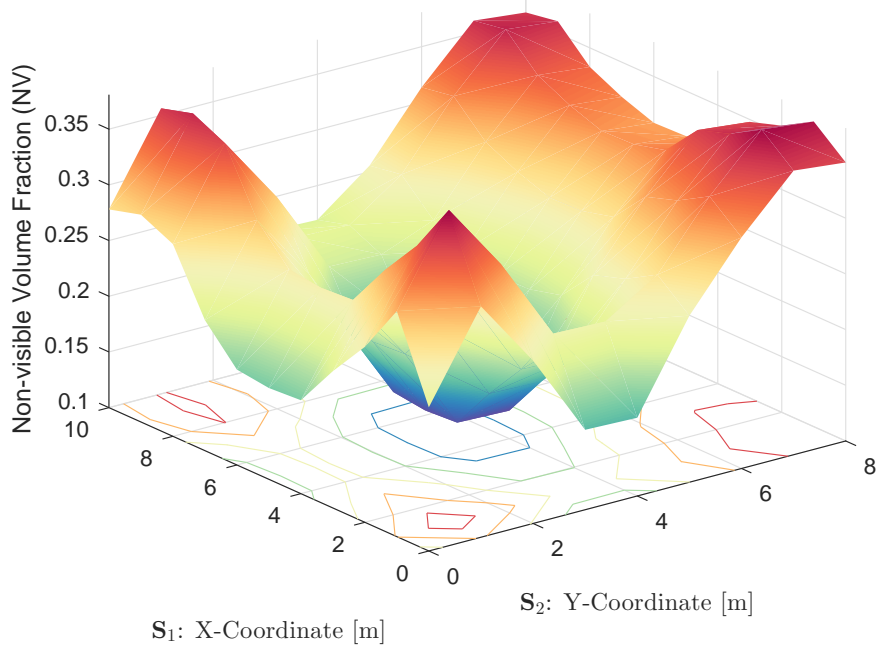

Fig. 4. Cost Function associated with the use case presented in Fig. 10. Varying only 2 out of the 20 optimization variables illustrates the nonconvexity of the cost function.

objective functions and the overall objective function for the minimax problem is not concave-convex. As an example, consider Fig. 4, which presents the objective function $(N V)$ for the upper level problem for the use case presented in Fig. 10. The occluder locations are fixed as in Fig. 10 and only two out of the twenty optimization parameters are varied to illustrate non-convexity of the objective function. It can be observed that the optimization problem is likely to encounter multiple local minima and regions with zero gradient.

Due to these characteristics, moving away from classical gradient-based optimization methods seems appropriate and the derivative-free pattern search algorithm [34] is utilized for obtaining the optimal solution for both levels in (17). Although a global optimum cannot be guaranteed in this work since pattern search algorithms require a continuously differentiable objective function to establish global convergence [35], the multi-start method is utilized to obtain trustworthy solutions. Varying the initial condition for the optimization problem and running the algorithm multiple times led to similar optimal solutions. Additionally, in lower dimensional cases, the optimal solution from pattern search is checked against an exhaustive search solution and is found to concur.

\section{VALIDATION}

This section provides a numerical validation of the robust optimization methodology for sensor planning described in Section III. First, an example is presented which illustrates the computation of the non-visible volume fraction for a given scenario. Further, the same example is utilized for validation of the robust optimization method.

\section{A. Illustrative Example - Visibility Model}

Consider the scenario presented in Fig. 5. A single static occluder $\mathbf{O}$ is located in the workspace $\mathcal{W}$ and a sensor $\mathbf{S}$ is installed on a wall in the room to capture the workspace visibility. The pyramid of vision $(\mathcal{P V})$ is illustrated in green. 


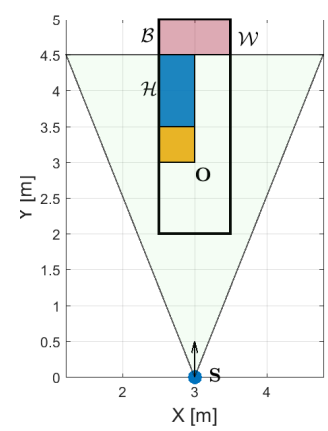

(a) Top View (2D)

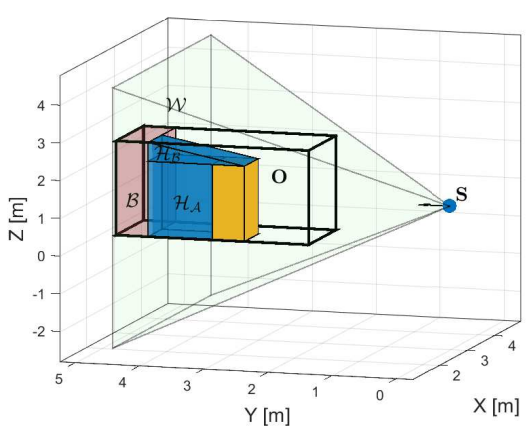

(b) Perspective View (3D)
Fig. 5. Validation Example - Visibility Model

TABLE I

SimUlation PARAMETERS - VALIDATION SCENARIO

\begin{tabular}{lll}
\hline Element & Parameter & Value [Unit] \\
\hline Workspace $(\mathcal{W})$ & Size & $1 \times 3 \times 2.5\left[\mathrm{~m}^{3}\right]$ \\
Occluder $(\mathbf{O})$ & Size & $0.5 \times 0.5 \times 2\left[\mathrm{~m}^{3}\right]$ \\
Sensor $(\mathbf{S})$ & Location & $(3,0,1)[\mathrm{m}]$ \\
& Horizontal FOV $(\alpha)$ & $35\left[^{\circ}\right]$ \\
& Vertical FOV $(\beta)$ & $75\left[^{\circ}\right]$ \\
& Maximum Range $\left(R_{\max }\right)$ & $4.5[\mathrm{~m}]$ \\
\hline
\end{tabular}

Simulation parameters of the elements are presented in Table I. The visibility analysis is carried out in MATLAB using the toolbox MPT 3.0 for polyhedral operations [36].

1) Blind Space: As observed in Fig. $5 \mathrm{a}, \mathcal{W}$ extends beyond $\mathcal{P V}$ as the sensor is limited by its maximum range. The sensor is thus not able to capture any part of the workspace beyond $4.5[\mathrm{~m}]$ in the Y-direction. The blind space is represented by the red polyhedron and is computed as

$$
\operatorname{Vol}(\mathcal{B})=1 \times 0.5 \times 2.5=1.25\left[m^{3}\right]
$$

2) Occluded Space: To generate the space occluded by an object $\mathbf{O}$ for a sensor $\mathbf{S}$, vectors connecting the apex and base of the sensor's FOV pyramid are constructed. These vectors pass through each of the vertices of the occluding object, creating a polyhedral space behind the occluder. Only the polyhedral space which is a part of the workspace is considered and the set difference of this space and the occluder gives the occluded volume.

The occluded volume $(\mathcal{H})$ is represented by the blue polyhedra in Fig. 5b. $\mathcal{H}$ is a combination of a cuboid $\mathcal{H}_{\mathcal{A}}$ (formed behind the occluder) and a right triangular prism $\mathcal{H}_{\mathcal{B}}$ (formed between occluder top and workspace ceiling). The occluded space is computed as

$$
\begin{aligned}
& \operatorname{Vol}\left(\mathcal{H}_{\mathcal{A}}\right)=0.5 \times 1 \times 2=1.0\left[\mathrm{~m}^{3}\right] \\
& \operatorname{Vol}\left(\mathcal{H}_{\mathcal{B}}\right)=\frac{1}{2} \times 0.5 \times 1.5 \times 0.5=0.1875\left[\mathrm{~m}^{3}\right] \\
& \operatorname{Vol}(\mathcal{H})=\operatorname{Vol}\left(\mathcal{H}_{\mathcal{A}}\right)+\operatorname{Vol}\left(\mathcal{H}_{\mathcal{B}}\right)=1.1875\left[\mathrm{~m}^{3}\right]
\end{aligned}
$$

3) Non-visible Volume Fraction: NV can be computed as follows:

$$
\begin{aligned}
& N V(\mathbf{O}, \mathbf{S})=\frac{\operatorname{Vol}(\mathcal{H} \cup \mathcal{B})}{\operatorname{Vol}(\mathcal{W} \backslash(\hat{\mathbf{O}} \cup \mathcal{R}))} \\
& N V(\mathbf{O}, \mathbf{S})=\frac{1.1875+1.25}{7.5-0.5} \\
& N V(\mathbf{O}, \mathbf{S})=0.3482
\end{aligned}
$$

\section{B. Validation - Robust Optimization for Sensor Planning}

The sensor planning method in (17) is validated based on the scenario in Fig. 5. The occluder $\mathbf{O}$ is constrained to move within the workspace, i.e, the constraint space $\mathcal{C}_{k}(k=1)$ is the polyhedral space $\mathcal{W}$ itself. For simplicity of presentation, ten different configurations of $\mathbf{O}$ are presented in Fig. 6a with the occluder shown at configurations 1,5 and 8. All possible locations of $\mathbf{O}$ form the occluder configuration set $\mathcal{O}$. To construct the set $\mathcal{S}$ of sensor configurations, the sensor Xcoordinate is considered as the parameter $p_{m}$ to be varied, with $m=1$. The constraints on this parameter are set as $l_{m}=2.5[\mathrm{~m}]$ and $u_{m}=3.5[\mathrm{~m}]$, with the $\mathrm{Y}$ and $\mathrm{Z}$ coordinate remaining fixed at $0[\mathrm{~m}]$ and $1[\mathrm{~m}]$ respectively.

Fig. $6 \mathrm{~b}$ is an illustrative representation of the optimization problem in (16). It presents the values of $N V$ over the entire design space for the scenario at hand with the black crosses representing the maximum $N V$ for a certain sensor configuration, i.e, the worst-case scenario. The minimum worst-case over all sensor configurations is represented by the black triangles. According to the optimization problem, the sensor configuration associated with this minimum worst-case is the optimal solution, i.e., $\mathbf{S}^{*}$. The sensor location associated with this minimum worst-case is $[3,0,1]$ and the associated occluder configurations are presented in Fig. 6c and Fig. 6d.

$\mathbf{S}=[3,0,1]$ implies that the sensor is placed central to the workspace width. Thus, for any position of the occluder, only one occluder face creates an occlusion of the workspace. For any other position of the sensor, there are always two occluder faces creating an occlusion and this occluded volume is higher than that created by a single face. Further, the associated occluder configurations from Fig. $6 \mathrm{c}$ and Fig. 6d are also intuitively expected since the occluder closest to the sensor cause the most amount of space to be occluded behind it.

The optimization problem in (16) is solved for this scenario in MATLAB using the pattern search algorithm with a mesh tolerance of $10^{-6}$ as the stopping criteria. An analytical justification for using mesh size as the stopping criteria is provided in [37]. The optimal solution provided by the algorithm $\left(\mathbf{S}^{*}=[3,0,1]\right)$ matches that of the exhaustive search approach described above, thus validating the global convergence guarantee of pattern search. Since the cost function (Fig. 6b) is continuously differentiable, the algorithm provides the global optimum for the optimization problem.

\section{Simulation And Results}

In this section, the applicability and potential of the robust optimization method is illustrated by focusing on a medical interventional X-ray system as the robotic manipulator. A description of the X-ray system along with the interventional 


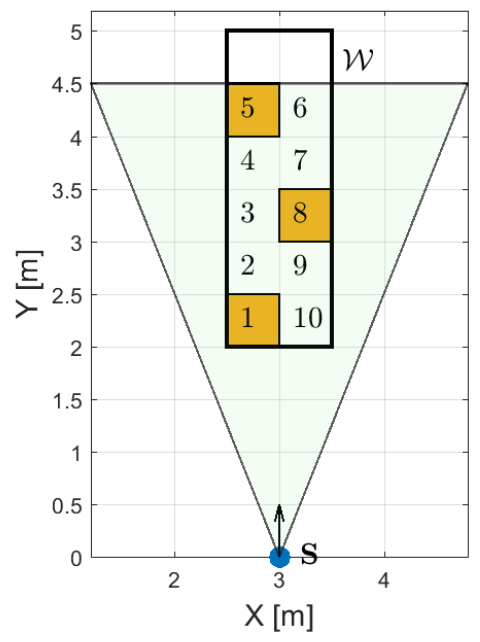

(a) Set of Occluder Configurations. The occluder $\mathbf{O}$ is shown at configurations 1,5 and 8 for illustration.

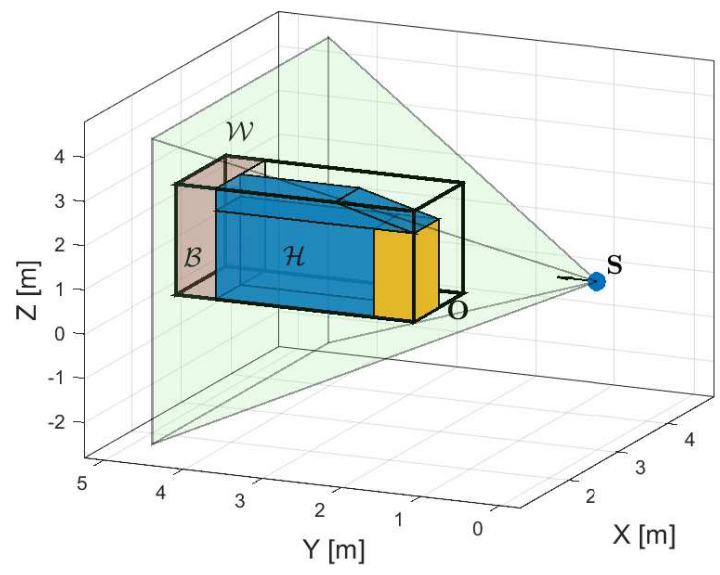

(c) Occluder Configuration - 1

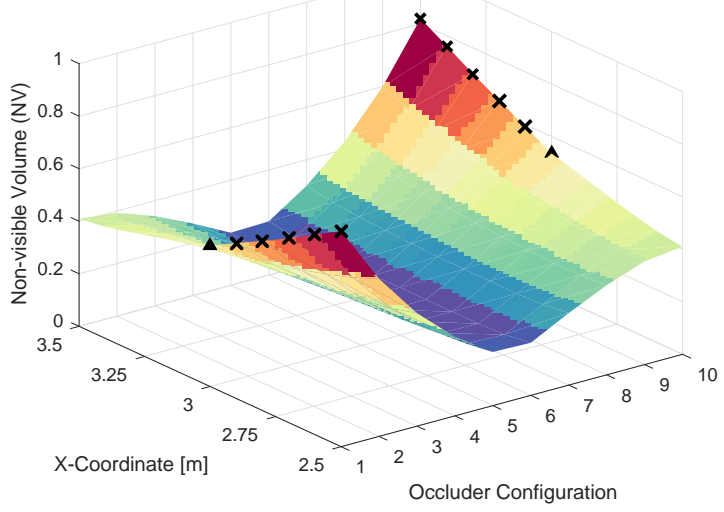

(b) Cost Function

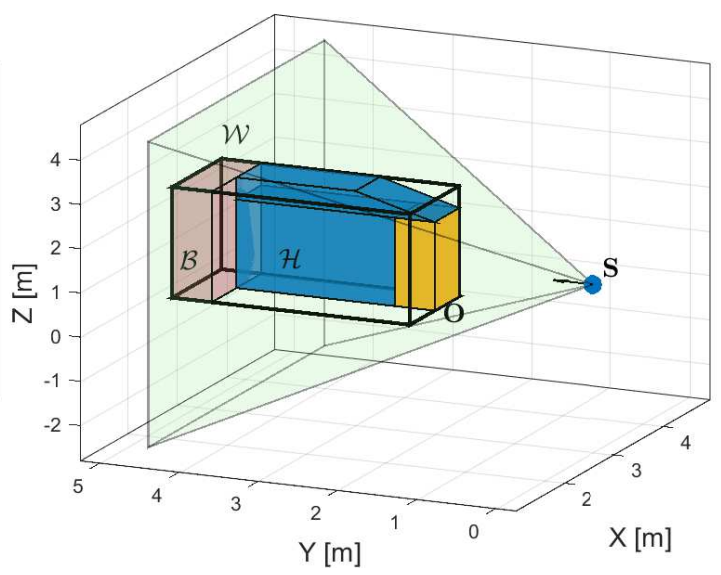

(d) Occluder Configuration - 10

Fig. 6. Illustrative representation of the robust optimization problem for the validation example: (a): Set of occluder configurations, (b): Values of $N V$ over the entire design space of the optimization problem, (c) \& (d): Occluder configurations for the optimal sensor configuration $\mathbf{S}=[3,0,1]$.

environment it operates in is provided. Results of the sensor planning approach are then presented for two medical use cases of the X-ray system.

\section{A. Interventional X-ray System and Simulation Environment}

Interventional X-ray systems are used in modern minimally invasive surgeries for providing high quality $3 \mathrm{D}$ images of the interior of the human body [38]. The system (Fig. 7) consists of a $\mathrm{C}$-shaped beam called the $\mathrm{C}$-arm, which mechanically connects the X-ray source and the flat detector. The $\mathrm{C}$-arm is connected to the so-called sleeve, which is in turn attached to an L-arm mounted to the ceiling or the floor. The L-arm can rotate around a vertical axis ( $Z$-axis), the $\mathrm{C}$-arm suspension can rotate around a horizontal axis attached to the $\mathrm{L}$-arm $(X$ axis), and the C-arm itself can be rotated around the axis defined by the intersection of the planes containing the L-arm and the suspension axes of rotation ( $Y$-axis). All three rotation axes meet at the isocenter of the system. Linear motion in $X$ and $Y$ direction can be provided by linear tracks mounted on the ceiling of the room [39]. Based on these movements, two primary use cases are considered for the X-ray system. The

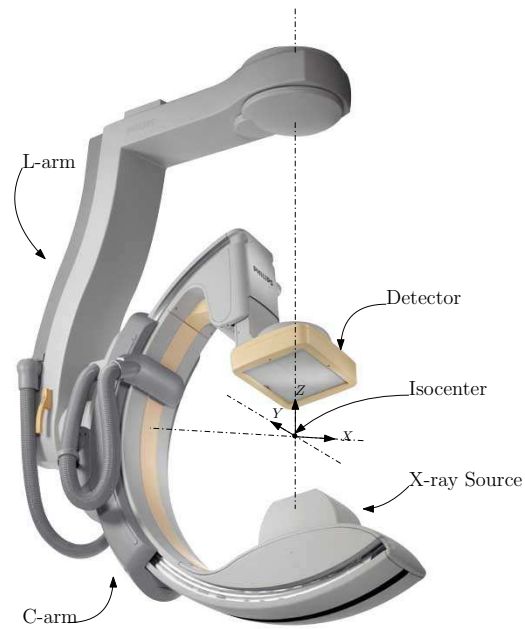

Fig. 7. Philips Allura FD20 Ceiling Suspended Dual Axis C-arc System.

first is an automated parking/docking movement in which the system is moved to a parking position in the room. This is done 


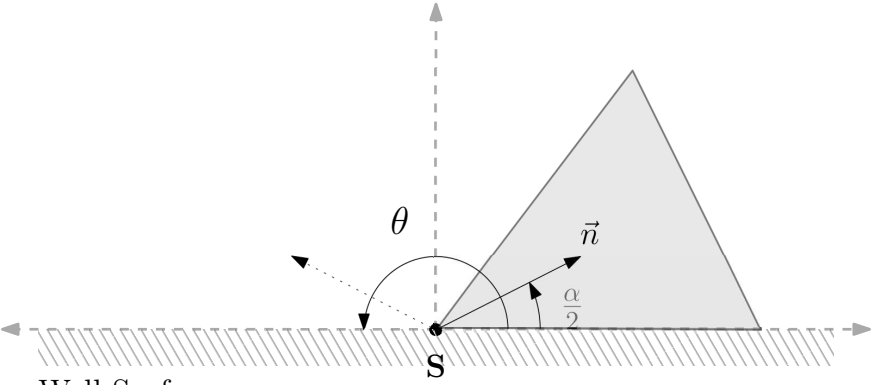

Wall Surface

(a) Constraint Satisfaction, $\theta=\alpha / 2$

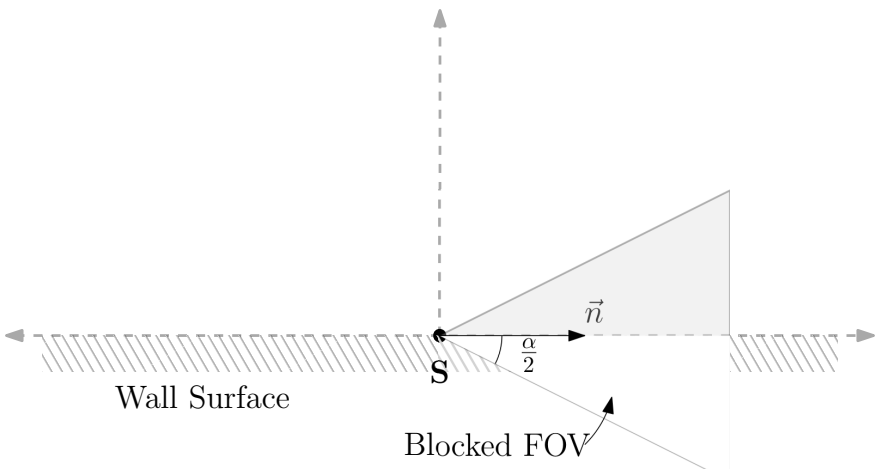

(b) Constraint Violation, $\theta=0^{\circ}$

Fig. 8. Schematic (top view) of the constraints on the pan angle $(\theta)$ of a sensor (S). Here, $\alpha$ is the horizontal FOV and $\vec{n}$ is the sensor normal.

using the linear motion in the $X-Y$ plane. The second use case is a medical scan termed 3D Rotational Angiography (3DRA) which utilizes the rotation of the $\mathrm{C}$-arm about the $X$-axis. The robust optimization method for sensor planning to detect collisions is evaluated using these use cases in a simulated interventional room which contains common elements for both the use cases. Apart from the system itself, the interventional room comprises of at least two moving humans, i.e, a doctor and nurse, a patient table and a monitor on which the X-ray images are viewed. The environment contains a fixed number of sensors $\left(n_{s}=4\right)$ which are constrained to be placed only on the four walls of the room and the ceiling. No sensors are placed on the floor so as to prevent the disruption of the work-flow. The pan $(\theta)$ and tilt $(\phi)$ angles for a sensor are constrained according to (18)

$$
\frac{\alpha}{2} \leq \theta<\pi-\frac{\alpha}{2} \quad \frac{\beta}{2} \leq \phi<\pi-\frac{\beta}{2}
$$

where $\alpha$ and $\beta$ are the horizontal and vertical FOV of the sensor. The constraints guarantee that the FOV of the sensors is never completely blocked by the wall (or ceiling) they are mounted on. This is schematically represented in Fig. 8a for the pan angle. The pan (or tilt) angle is considered as the angle formed between the sensor normal $(\vec{n})$ and wall (or ceiling) surface on which the sensor is mounted with the angle defined as positive counter-clockwise. If the pan angle value violates the constraints (18), the FOV would be occluded by the wall surface as represented in Fig. 8b with $\theta=0^{\circ}$.

It is important to note that a real-world interventional room contains more elements than the ones described, however, for
TABLE II

SIMULATION PARAMETERS

\begin{tabular}{lcc}
\hline Parameter & Symbol & Value \\
\hline Environment & $\mathcal{E}$ & Automated Parking $-10 \times 8 \times 4\left[\mathrm{~m}^{3}\right]$ \\
\cline { 3 - 3 } & & $3 D R A-8 \times 5 \times 4\left[\mathrm{~m}^{3}\right]$
\end{tabular}

Workspace

$\mathcal{W}$

$$
\begin{gathered}
\text { Automated Parking }-\mathbf{W}_{\mathbf{1}} \cup \mathbf{W}_{\mathbf{2}} \\
\mathbf{W}_{\mathbf{1}}-3 \times 4.1 \times 3.8\left[\mathrm{~m}^{3}\right] \\
\mathbf{W}_{\mathbf{2}}-8.5 \times 1.1 \times 3.8\left[\mathrm{~m}^{3}\right] \\
3 D R A-2.2 \times 2.3 \times 2.5\left[\mathrm{~m}^{3}\right]
\end{gathered}
$$

\begin{tabular}{|c|c|c|}
\hline \multicolumn{3}{|c|}{ Occluding Objects } \\
\hline Number of Occluders & $n_{o}$ & 5 \\
\hline X-ray System - Dynamic & $\mathcal{R}$ & Dimensions presented in Fig. 9 \\
\hline Doctor - Dynamic & $\mathrm{O}_{1}$ & $0.75 \times 0.75 \times 1.8\left[\mathrm{~m}^{3}\right]$ \\
\hline Nurse - Dynamic & $\mathrm{O}_{2}$ & $0.75 \times 0.75 \times 1.8\left[\mathrm{~m}^{3}\right]$ \\
\hline Table - Static & $\mathrm{O}_{3}$ & Base $-0.5 \times 0.5 \times 1.3\left[\mathrm{~m}^{3}\right]$ \\
\hline Monitor - Static & $\mathrm{O}_{4}$ & $\begin{array}{l}\text { Table Top }-3 \times 0.4 \times 0.2\left[\mathrm{~m}^{3}\right] \\
\quad 2 \times 0.5 \times 1\left[\mathrm{~m}^{3}\right]\end{array}$ \\
\hline \multicolumn{3}{|c|}{ Sensors } \\
\hline Number of Sensors & $n_{s}$ & 4 \\
\hline Sensor Parameters & $n_{p}$ & 5 \\
\hline Horizontal FOV & $\alpha$ & $70^{\circ}$ \\
\hline Vertical FOV & $\beta$ & $60^{\circ}$ \\
\hline Maximum Range & $R_{\max }$ & $4.5 \mathrm{~m}$ \\
\hline
\end{tabular}

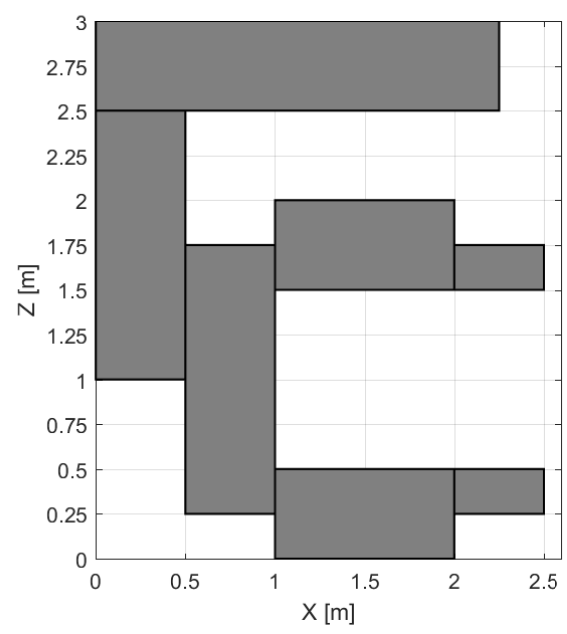

Fig. 9. Polyhedron block structure of the X-ray system in Fig. 7. The dimensions in $\mathrm{X}$ and $\mathrm{Z}$ directions are presented. The dimension of each block in $\mathrm{Y}$ direction is $0.7[\mathrm{~m}]$.

the sake of simplicity other entities such as medical carts, operation lights, additional medical staff, etc. are omitted. The parameters for various elements used during simulation are presented in Table II.

\section{B. Use Case 1: Automated Parking}

The X-ray system occupies a significant amount of space around the patient table. Once imaging of the patient body is complete, it is required to move the system away to free up space for the doctors to continue with the surgery. For this 


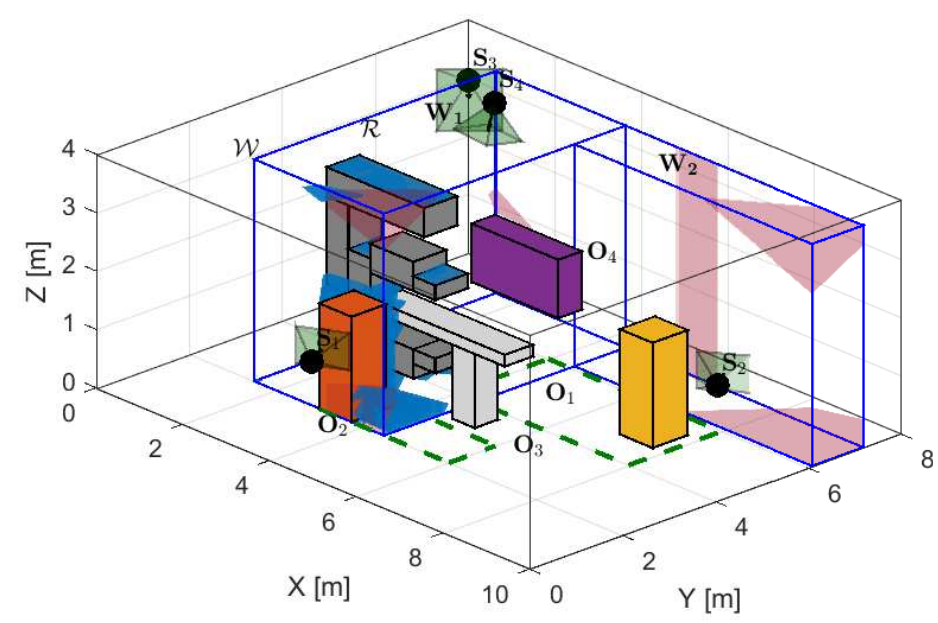

(a) Perspective View (3D)

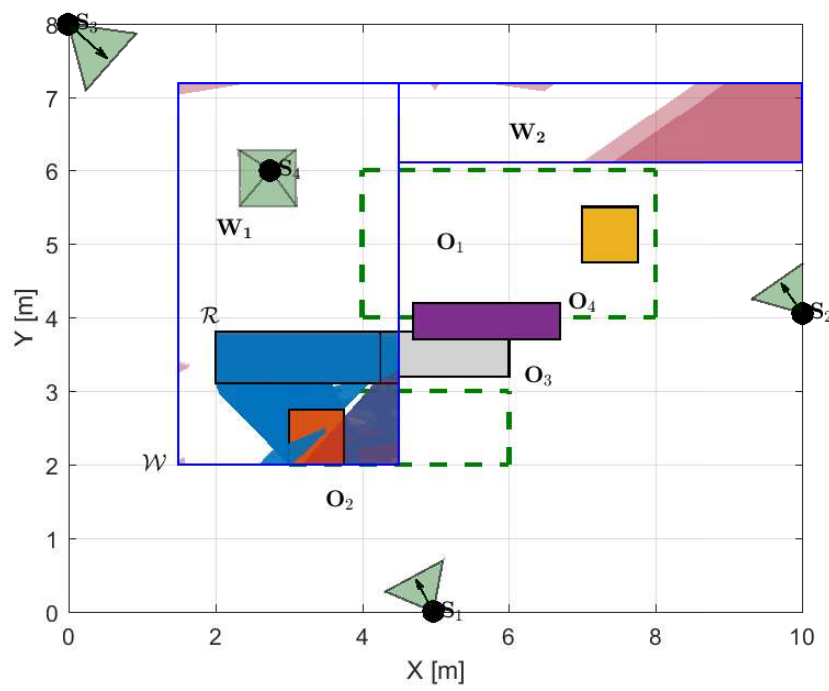

(b) Top View (2D)

Fig. 10. Optimal sensor configuration $\hat{\mathbf{S}}_{\mathrm{AP}}^{*}$ and associated occluder configuration for Automated Parking: $N V=0.09$, Blind Space $(\mathcal{B})$ - Red, Occluded Space $(\mathcal{H})$ - Blue, Robot $(\mathcal{R})$ - Gray, Doctor $\left(\mathbf{O}_{\mathbf{1}}\right)$ - Yellow, Nurse $\left(\mathbf{O}_{\mathbf{2}}\right)$ - Orange, Table $\left(\mathbf{O}_{\mathbf{3}}\right)$ - Light Gray, Monitor $\left(\mathbf{O}_{4}\right)$ - Purple. The FOV (not to scale) of each sensor is shown for illustrative purposes.

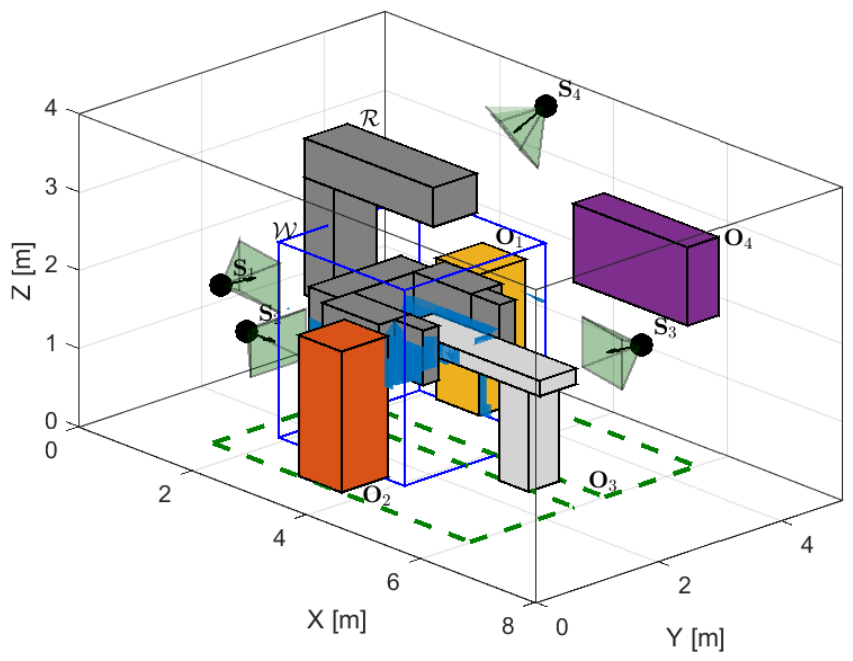

(a) Perspective View (3D)

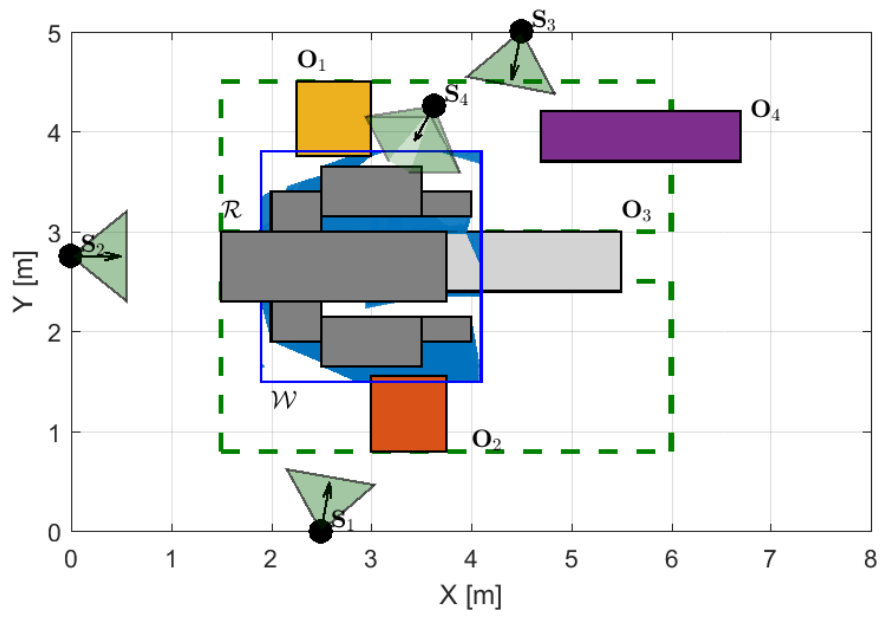

(b) Top View (2D)

Fig. 11. Optimal sensor configuration $\hat{\mathbf{S}}_{3 \mathrm{D}}^{*}$ and associated occluder configuration for $3 D R A: N V=0.02$, Blind Space $(\mathcal{B})$ - None, Occluded Space $(\mathcal{H})$ Blue, Robot $(\mathcal{R})$ - Gray, Doctor $\left(\mathbf{O}_{1}\right)$ - Yellow, Nurse $\left(\mathbf{O}_{2}\right)$ - Orange, Table $\left(\mathbf{O}_{3}\right)$ - Light Gray, Monitor $\left(\mathbf{O}_{4}\right)$ - Purple. The FOV (not to scale) of each sensor is shown for illustrative purposes.

purpose, the system has a dedicated spot in the interventional room where it can be "parked". Collision-free parking of the system from the table to the parking spot necessitates sensor planning to efficiently detect possible collisions. This problem is analogous to the collision-free motion of mobile robots.

Figure 10 presents the optimal solution to the minimax sensor planning problem for the automated parking use case. The parking motion is simulated by movement of the system $(\mathcal{R})$ from the patient table to the parking spot by considering lateral movement in $\mathbf{W}_{1}$ and longitudinal movement in $\mathbf{W}_{2}$. The dynamic occluders $\mathbf{O}_{1}$ and $\mathbf{O}_{2}$ are constrained to move in the polyhedral spaces $\left(\mathcal{C}_{1}\right.$ and $\left.\mathcal{C}_{2}\right)$ represented by the green boxes (dashed) with dimensions $4 \times 2 \times 1.8\left[\mathrm{~m}^{3}\right]$ for $\mathbf{O}_{1}$ and $3 \times 1 \times 1.8\left[\mathrm{~m}^{3}\right]$ for $\mathbf{O}_{2}$. The location of static occluders $\mathbf{O}_{3}$ and $\mathrm{O}_{4}$ remains constant and their respective constraints $\mathcal{C}_{3}$ and $\mathcal{C}_{4}$ are the polyhedral occluders themselves.

Sensors $\mathbf{S}_{1}, \mathbf{S}_{2}$ and $\mathbf{S}_{3}$ are constrained to the walls while $\mathbf{S}_{4}$ is attached to the ceiling. Varying the coordinates of the sensors according to respective wall (or ceiling) dimensions and the pan and tilt angles according to (18) creates the set of sensor configurations. 
TABLE III

OPTIMAL SENSOR CONFIGURATIONS

\begin{tabular}{ccccccc}
\hline Scenario & Sensor & $\mathrm{X}[\mathrm{m}]$ & $\mathrm{Y}[\mathrm{m}]$ & $\mathrm{Z}[\mathrm{m}]$ & $\theta\left[{ }^{\circ}\right]$ & $\phi\left[^{\circ}\right]$ \\
\hline Automated Parking & $\mathbf{S}_{1}$ & 5.00 & 0 & 2.00 & 118 & 0 \\
$\hat{\mathbf{S}}_{\text {AP }}^{*}$ & $\mathbf{S}_{2}$ & 10.00 & 4.05 & 1.96 & 35 & 0 \\
& $\mathbf{S}_{3}$ & 0 & 8.00 & 2.93 & 45 & 0 \\
& $\mathbf{S}_{4}$ & 2.75 & 6.00 & 4.00 & 78 & 93 \\
\hline 3DRA & $\mathbf{S}_{1}$ & 2.50 & 0 & 2.50 & 80 & 0 \\
$\hat{\mathbf{S}}_{3 \mathrm{D}}^{*}$ & $\mathbf{S}_{2}$ & 0 & 2.75 & 0.50 & 90 & 0 \\
& $\mathbf{S}_{3}$ & 4.50 & 5.00 & 1.00 & 79 & 0 \\
& $\mathbf{S}_{4}$ & 3.62 & 4.25 & 4.00 & 46 & 58 \\
\hline
\end{tabular}

\section{Use Case 2: 3D Rotational Angiography (3DRA)}

Rotational Angiography is an image acquisition technique used to obtain a three dimensional image of the patient's heart. The X-ray system is positioned at the end of the patient table and the $\mathrm{C}$-arm makes an angular rotation about the $\mathrm{X}$-axis, acquiring X-ray images of the patient's heart during the entire rotation. During this scan, it is imperative that the C-arm does not collide with the patient or the table, thus necessitating accurate workspace detection

The 3DRA scenario along with the optimal solution to (17) is presented in Fig. 11. The C-arm of the X-ray system $(\mathcal{R})$ makes a $90^{\circ}$ rotation about the $\mathrm{X}$-axis with the $\mathrm{C}$-arm at $90^{\circ}$ shown in Fig. 11 and at $0^{\circ}$ shown in Fig. 10. The dynamic occluder constraints $\left(\mathcal{C}_{1}\right.$ and $\left.\mathcal{C}_{2}\right)$ are represented by the green boxes (dashed) of dimensions $4.5 \times 1.5 \times 1.8\left[\mathrm{~m}^{3}\right]$ for $\mathbf{O}_{1}$ and $4.5 \times 1.7 \times 1.8\left[\mathrm{~m}^{3}\right]$ for $\mathbf{O}_{2}$. The constraints for the static occluders are as described in the previous use case.

Similar to the automated parking use case, $\mathbf{S}_{1}, \mathbf{S}_{2}$ and $\mathbf{S}_{3}$ are constrained to the walls while $\mathbf{S}_{4}$ is attached to the ceiling. Constructing various sensor configurations is carried out in the same manner as before.

\section{Discussion}

Sensor planning for the two use cases is carried out using the minimax optimization problem in (17) and the optimal solutions are presented in Table III. The associated worstcase occluder configurations along with workspace visibility are presented in Fig. 10 and Fig. 11. The results provide the following insights:

1) The solution is obtained without an explicit probability distribution while still accounting for random occlusions. Further, since the minimax problem considers optimization over the complete set of occluder configurations, the optimal solution accounts for critical scenarios.

2) The proposed optimization approach provides a guarantee on the performance of the optimal sensor configuration. Firstly, it is guaranteed that the worst-case $N V$ for $\hat{\mathbf{S}}^{*}$ is the lowest compared to all other sensor configurations. Further, regardless of occluder configuration, the visibility for this optimal solution $\hat{\mathbf{S}}^{*}$ will always be higher than the worst-case $N V$.

3) For the automated parking use case, the worst-case workspace visibility occurs at the beginning of the parking motion, i.e., when the $\mathrm{C}$-arm is at the patient table. The occlusion of the workspace (blue) exists in the region between $\mathcal{R}$ and $\mathrm{O}_{2}$ since the nurse occludes sensor $\mathbf{S}_{1}$ and $\mathrm{C}$-arm occludes $\mathbf{S}_{4}$. It is interesting to note the shape of the occluded volume since the shape and dimensions are such that no object can completely occupy this space. The volume along the Y-axis is not wide enough for a complete object to envelop it. Moreover, if an additional object exists next to $\mathrm{O}_{2}$ then only part of the object will be invisible while most of it can still be detected and a potential collision can be avoided. This suggests that worst-case visibility is not always most critical for collisions.

4) The worst-case scenario for 3DRA shows most of the occluded space to be around the patient table due to the occlusion by the system itself. Since the safety of the patient is of prime importance, the optimal sensor configuration in Fig. 11 might not cater to that. Contrary to the parking use case, the worst-case scenario for 3DRA is also a critical collision scenario. Although increase in the number of sensors could be helpful, placing a sensor on the $\mathrm{C}$-arm itself could prove to be more valuable to provide visibility of the critical space.

Apart from generating a sensor configuration for the use cases, the visibility analysis along with the optimization results provide insights to the user on the impact of the worst case situation. These insights can further be used to assess the criticality of the worst-case scenarios and account for them during medical procedures.

\section{E. Impact of Varying Number of Occluders}

Robust optimization for sensor planning is based on an initial estimate of occluders $\left(n_{o}\right)$ present in the environment. It is thus important to discuss the effect of $n_{o}$ deviating from the initial estimated value. Consider the 3DRA use case in Fig. 11 with the optimal solution $\hat{\mathbf{S}}_{3 \mathrm{D}}^{*}$ for $n_{o}=5$ occluders (Table III). An extra object $\mathrm{O}_{5}$ (e.g. a small cart) is now added to the environment and is constrained to exist in polyhedral space $\mathcal{C}_{5}$ represented by the red dashed boxes (Fig. 12a). The lower-level optimization problem in (17) is solved for this situation with $\hat{\mathbf{S}}=\hat{\mathbf{S}}_{3 \mathrm{D}}^{*}$. The occluder configuration $\left(\hat{\mathbf{O}}_{+}^{*}\right)$ which maximizes the cost function $N V(\hat{\mathbf{O}}, \hat{\mathbf{S}})$ is presented in Fig. 12a with $N V=0.4$ which is much larger than $N V$ $=0.09$ for $\hat{\mathbf{S}}_{3 \mathrm{D}}^{*}$ with $n_{o}=5$. This implies that $\hat{\mathbf{S}}_{3 \mathrm{D}}^{*}$ does not adapt well to the additional occluder and the sensor configuration needs to be adjusted in order to reduce $N V$. To this end, the upper level optimization problem in (17) is solved with occluder configuration $\mathbf{O}=\hat{\mathbf{O}}_{+}^{*}$ and the adjusted optimal sensor configuration obtained $\left(\hat{\mathbf{S}}_{+}^{*}\right)$ is compared with the previous optimum in Table IV (adjusted values presented in blue). As observed in Fig. $12 \mathrm{~b}, \hat{\mathbf{S}}_{+}^{*}$ reduces $N V$ to 0.17 with parameters being adjusted for all 4 sensors.

In general, the optimal sensor configuration needs to be adjusted when $n_{o}$ deviates from the initial estimate. Similarly, a change in occluder size, shape and location constraints will also lead to solving the optimization problem (17) again in order to find a new optimum. 
TABLE IV

IMPACT OF VARYING OCCLUDERS FOR 3DRA: COMPARISON OF OPTIMAL SENSOR CONFIGURATIONS

\begin{tabular}{lcccccc}
\hline Scenario & Sensor & $\mathrm{X}[\mathrm{m}]$ & $\mathrm{Y}[\mathrm{m}]$ & $\mathrm{Z}[\mathrm{m}]$ & $\theta\left[{ }^{\circ}\right]$ & $\phi\left[^{\circ}\right]$ \\
\hline$\hat{\mathbf{S}}_{3 \mathrm{D}}^{*}, n_{o}=5$ & $\mathbf{S}_{1}$ & 2.50 & 0 & 2.50 & 80 & 0 \\
(Fig. 11) & $\mathbf{S}_{2}$ & 0 & 2.75 & 0.50 & 90 & 0 \\
& $\mathbf{S}_{3}$ & 4.50 & 5.00 & 1.00 & 79 & 0 \\
& $\mathbf{S}_{4}$ & 3.62 & 4.25 & 4.00 & 46 & 58 \\
\hline$\hat{\mathbf{S}}_{+}^{*}, n_{O}=6$ & $\mathbf{S}_{1}$ & 2.50 & 0 & 2.50 & $\mathbf{6 5}$ & 0 \\
(Fig. 12b) & $\mathbf{S}_{2}$ & 0 & $\mathbf{3 . 7 5}$ & 0.50 & $\mathbf{7 4}$ & 0 \\
& $\mathbf{S}_{3}$ & $\mathbf{2 . 5 0}$ & 5.00 & 1.00 & 79 & 0 \\
& $\mathbf{S}_{4}$ & 3.62 & 4.25 & 4.00 & $\mathbf{7 2}$ & $\mathbf{7 0}$ \\
\hline
\end{tabular}

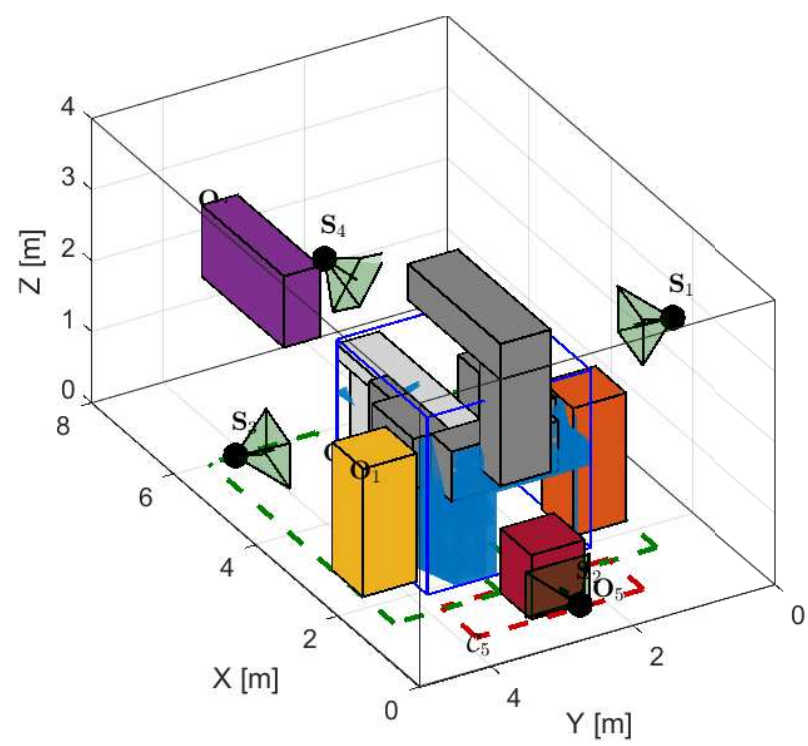

(a) Worst-case Occluder Configuration $\hat{\mathbf{O}}_{+}^{*}$ for $\hat{\mathbf{S}}_{3 \mathrm{D}}^{*}, N V=0.4$

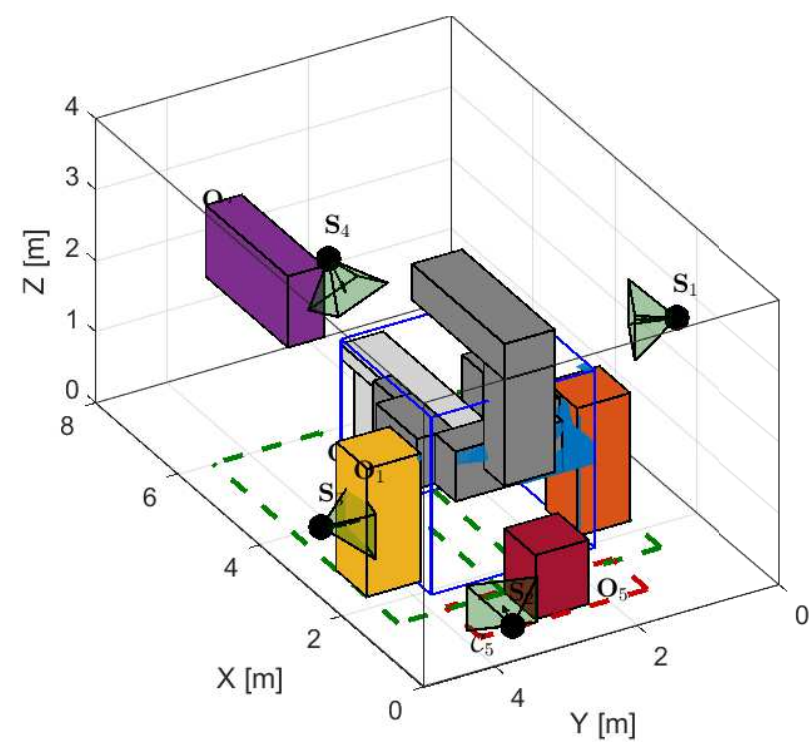

(b) Optimal Sensor Configuration $\hat{\mathbf{S}}_{+}^{*}$ for $\hat{\mathbf{O}}_{+}^{*}, N V=0.17$

Fig. 12. Impact of Varying $n_{o}$ for $3 D R A$ : Blind Space $(\mathcal{B})$ - None, Occluded Space $(\mathcal{H})$ - Blue, Robot $(\mathcal{R})$ - Gray, Doctor $\left(\mathbf{O}_{1}\right)$ - Yellow, Nurse $\left(\mathbf{O}_{2}\right)$ Orange, Table $\left(\mathbf{O}_{3}\right)$ - Light Gray, Monitor $\left(\mathbf{O}_{4}\right)$ - Purple, Cart $\left(\mathbf{O}_{5}\right)$ - Red.

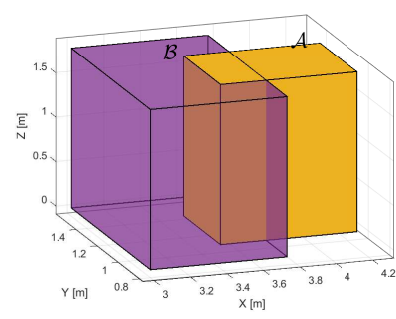

(a) Before Set-difference

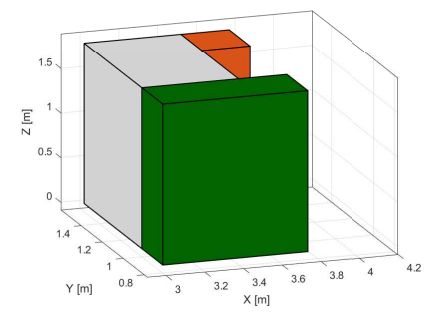

(b) After Set-difference $\mathcal{B} \backslash \mathcal{A}$
Fig. 13. Illustration - Set-difference $(\backslash$ ) of polyhedra $\mathcal{A}$ (yellow) and $\mathcal{B}$ (purple)

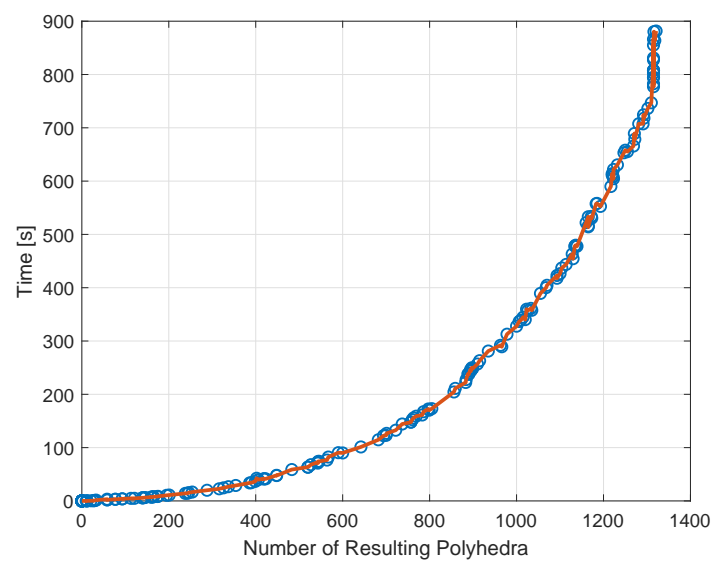

Fig. 14. CPU time required to compute number of polyhedra $(|\mathcal{H}|)$ as the output of a set-difference operation $\mathcal{H}=\mathcal{X} \backslash \mathcal{Z}$

\section{F. Computational Complexity}

The highest computationally intensive operation while calculating the objective function $N V$ in (17) is the set-difference operation $(\mathcal{B} \backslash \mathcal{A})$ on multiple polyhedra. In MPT 3.0, the setdifference operation of two convex polyhedra does not result in a single non-convex polyhedron, but in multiple convex polyhedra depending on the number of clipping planes involved in the set-difference. Consider the example in Fig. 13 where $\mathcal{B} \backslash \mathcal{A}$ (Fig. 13a) leads to the creation of 3 polyhedra (Fig. 13b). As a result, in (14), increase in $|\mathcal{X}|$ and $|\mathcal{Z}|$ leads to a large number of resulting polyhedra in the output, i.e., large $|\mathcal{H}|^{1}$. Fig. 14 presents the computation time required as a function of output polyhedra generated during a set-difference operation in MPT 3.0. The results illustrate exponential time complexity as the number of output polyhedra $|\mathcal{H}|$ becomes large. Since evaluating $N V$ is directly dependent on computing $\mathcal{H}$, the time complexity associated with the minimax optimization problem is also exponential.

Exponential time complexity hampers the overall computational efficiency of the minimax optimization problem. Relying on more computationally efficient algorithms for polyhedral operations such as [40] could prove to be more appropriate to ease computational demands. 


\section{CONCLUSION}

This work addressed the problem of optimal sensor planning for target workspace detection in the presence of random occlusions. Treating random occlusions as uncertainty in the sensor planning problem, a robust optimization (minimax) approach is proposed to generate optimal sensor configurations. The approach evaluates occluder configurations that potentially maximize the occluded volume in a target space and computes sensor configurations that guard against these scenarios to provide visibility guarantees.

The approach is evaluated in a 3D simulation environment with a medical X-ray system as the robotic manipulator and elements in the hospital room as dynamic occluders. The result is free of probabilistic distributions and no apriori assumptions are made about the probability of location of occluders. Further, since the optimal solution guards against the worstcase scenario, a certain visibility of the target workspace is always guaranteed regardless of occluder positions. The visibility analysis in three dimensions also provide insights to the user on the impact of the worst-case situation on workspace visibility, thus opening up a broader perspective on sensor placement in dynamic and uncertain environments.

\section{REFERENCES}

[1] A. A. Altahir, V. S. Asirvadam, N. H. B. Hamid, P. Sebastian, N. B. Saad, R. B. Ibrahim, and S. C. Dass, "Optimizing visual surveillance sensor coverage using dynamic programming," IEEE Sensors Journal, vol. 17, no. 11, pp. 3398-3405, 2017.

[2] J. Zhao, R. Yoshida, S. C. S. Cheung, and D. Haws, "Approximate techniques in solving optimal camera placement problems," International Journal of Distributed Sensor Networks, vol. 9, no. 11, 2013, Art. ID 241913.

[3] Y. Morsly, N. Aouf, M. S. Djouadi, and M. Richardson, "Particle swarm optimization inspired probability algorithm for optimal camera network placement," IEEE Sensors Journal, vol. 12, no. 5, pp. 1402-1412, 2012.

[4] A. Aissaoui, A. Ouafi, P. Pudlo, C. Gillet, Z.-E. Baarir, and A. TalebAhmed, "Designing a camera placement assistance system for human motion capture based on a guided genetic algorithm," Virtual Reality, vol. 22 , no. 1 , pp. 13-23, 2018.

[5] A. Mavrinac and X. Chen, "Modeling coverage in camera networks: A survey," International Journal of Computer Vision, vol. 101, no. 1, pp. 205-226, 2013

[6] S. Jun, T.-W. Chang, H. Jeong, and S. Lee, "Camera placement in smart cities for maximizing weighted coverage with budget limit," IEEE Sensors Journal, vol. 17, no. 23, pp. 7694-7703, 2017.

[7] S. A. Mostafavi and M. Dehghan, "Optimal visual sensor placement for coverage based on target location profile," Ad Hoc Networks, vol. 9, no. 4, pp. 528-541, 2011.

[8] H. Hu and M. Brady, "A Bayesian approach to real-time obstacle avoidance for a mobile robot," Autonomous Robots, vol. 1, no. 1, pp. 69-92, 1994.

[9] S. Verdu and H. Poor, "On minimax robustness: A general approach and applications," IEEE Transactions on Information Theory, vol. 30, no. 2, pp. 328-340, 1984.

[10] K. A. Tarabanis, P. K. Allen, and R. Y. Tsai, "A survey of sensor planning in computer vision," IEEE Transactions on Robotics and Automation, vol. 11, no. 1, pp. 86-104, 1995.

[11] J. O'Rourke, Art gallery theorems and algorithms. Oxford University Press Oxford, 1987.

[12] K. R. Konda, N. Conci, and F. De Natale, "Global coverage maximization in PTZ-camera networks based on visual quality assessment," IEEE Sensors Journal, vol. 16, no. 16, pp. 6317-6332, 2016.

[13] M. Rebai, M. le Berre, F. Hnaien, and H. Snoussi, "Exact biobjective optimization methods for camera coverage problem in three-dimensional areas," IEEE Sensors Journal, vol. 16, no. 9, pp. 3323-3331, 2016.

[14] A. Mittal and L. S. Davis, "A general method for sensor planning in multi-sensor systems: Extension to random occlusion," International Journal of Computer Vision, vol. 76, no. 1, pp. 31-52, 2008.
[15] X. Chen and J. Davis, "An occlusion metric for selecting robust camera configurations," Machine Vision and Applications, vol. 19, no. 4, pp. 217-222, 2008

[16] R. Bodor, A. Drenner, P. Schrater, and N. Papanikolopoulos, "Optimal camera placement for automated surveillance tasks," Journal of Intelligent \& Robotic Systems, vol. 50, no. 3, pp. 257-295, 2007.

[17] F. Flacco and A. De Luca, "Multiple depth/presence sensors: Integration and optimal placement for human/robot coexistence," in 2010 IEEE International Conference on Robotics and Automation (ICRA). IEEE, 2010, pp. 3916-3923.

[18] A. Shapiro and A. Kleywegt, "Minimax analysis of stochastic problems," Optimization Methods and Software, vol. 17, no. 3, pp. 523-542, 2002.

[19] C. Yang, Z. Lu, and Z. Yang, "Robust optimal sensor placement for uncertain structures with interval parameters," IEEE Sensors Journal, vol. 18, no. 5, pp. 2031-2041, 2018.

[20] J. O. Berger, Statistical decision theory and Bayesian analysis. Springer, 1985.

[21] T. Başar and P. Bernhard, H-infinity optimal control and related minimax design problems: A dynamic game approach. Birkhäuser Boston, 1991.

[22] J. Marzat, E. Walter, and H. Piet-Lahanier, "Worst-case global optimization of black-box functions through Kriging and relaxation," Journal of Global Optimization, vol. 55, no. 4, pp. 707-727, 2013.

[23] O. A. Vanli, C. Zhang, A. Nguyen, and B. Wang, "A minimax sensor placement approach for damage detection in composite structures," Journal of Intelligent Material Systems and Structures, vol. 23, no. 8, pp. 919-932, 2012.

[24] B. Liu, O. Dousse, P. Nain, and D. Towsley, "Dynamic coverage of mobile sensor networks," IEEE Transactions on Parallel and Distributed systems, vol. 24, no. 2, pp. 301-311, 2013.

[25] J. W. Herrmann, "A genetic algorithm for minimax optimization problems," in Proceedings of the 1999 Congress on Evolutionary Computation, vol. 2. IEEE, 1999, pp. 1099-1103.

[26] A. Bemporad, F. Borrelli, and M. Morari, "Min-max control of constrained uncertain discrete-time linear systems," IEEE Transactions on Automatic Control, vol. 48, no. 9, pp. 1600-1606, 2003.

[27] J. Liu, S. Sridharan, and C. Fookes, "Recent advances in camera planning for large area surveillance: A comprehensive review," $A C M$ Comput. Surv., vol. 49, no. 1, pp. 6:1-6:37, May 2016.

[28] A. A. Altahir, V. S. Asirvadam, N. H. B. Hamid, P. Sebastian, N. B. Saad, R. B. Ibrahim, and S. C. Dass, "Optimizing visual sensor coverage overlaps for multiview surveillance systems," IEEE Sensors Journal, vol. 18, no. 11, pp. 4544-4552, 2018.

[29] G. M. Ziegler, Lectures on polytopes. Springer, 1995.

[30] S. M. LaValle, Planning Algorithms. Cambridge University Press, 2006.

[31] K. Tarabanis, R. Y. Tsai, and A. Kaul, "Computing occlusion-free viewpoints," IEEE Transactions on Pattern Analysis and Machine Intelligence, vol. 18, no. 3, pp. 279-292, 1996.

[32] B. Rustem, S. Žaković, and P. Parpas, "An interior point algorithm for continuous minimax: implementation and computation," Optimisation Methods \& Software, vol. 23, no. 6, pp. 911-928, 2008.

[33] R. T. Rockafellar, Convex analysis. Princeton university press, 1997.

[34] R. Hooke and T. A. Jeeves, "'Direct Search' solution of numerical and statistical problems," Journal of the ACM (JACM), vol. 8, no. 2, pp. 212-229, 1961

[35] V. Torczon, "On the convergence of pattern search algorithms," SIAM Journal on optimization, vol. 7, no. 1, pp. 1-25, 1997.

[36] M. Herceg, M. Kvasnica, C. Jones, and M. Morari, "Multi-Parametric Toolbox 3.0," in Proc. of the European Control Conference, 2013, pp. 502-510.

[37] E. D. Dolan, R. M. Lewis, and V. Torczon, "On the local convergence of pattern search," SIAM Journal on Optimization, vol. 14, no. 2, pp. 567-583, 2003.

[38] R. van der Maas, J. Dries, B. de Jager, and M. Steinbuch, "Model-based geometric calibration for medical X-ray systems," Medical Physics, vol. 42, no. 11, pp. 6170-6182, 2015.

[39] R. Fahrig, J. Starman, E. Girard, A. Al-Ahmad, H. Gao, N. Kothary, and A. Ganguly, "C-arm CT in the interventional suite: Current status and future directions," in Cone Beam Computed Tomography, C. Shaw, Ed. CRC Press, 2014, ch. 14, pp. 199-221.

[40] D. Powell and T. Abel, "An exact general remeshing scheme applied to physically conservative voxelization," Journal of Computational Physics, vol. 297, pp. 340-356, 2015. 